\title{
Efficacy of Levamisole with Standard Care Treatment vs Standard Care in Clinical Presentations of Non-Hospitalized Patients with COVID-19: A Randomized Clinical Trial
}

Mohammad Hossein Asgardoon ( $\nabla$ mh_asgardoon@yahoo.com )

AJA University of Medical Sciences

Hamid Emadi koochak

Tehran University of Medical Science

Mohammad Hassan Kazemi-Galougahi

Aja University of Medical Sciences

Ali Zare Dehnavi

Tehran University of Medical Sciences

Behzad Khodaei

Tehran University of Medical Sciences

Atefeh Behkar

Tehran University of Medical Sciences

Ahmad Reza Dehpour

Tehran University of Medical Sciences

Hossein Khalili

Tehran University of Medical Science

Mohammad Aminianfar

AJA University of Medical Sciences

\section{Research Article}

Keywords: Levamisole, COVID-19, SARS-CoV2, Clinical status

Posted Date: November 15th, 2021

DOI: https://doi.org/10.21203/rs.3.rs-964097/v1

License: (c) (1) This work is licensed under a Creative Commons Attribution 4.0 International License.

Read Full License 


\section{Abstract \\ Introduction:}

Levamisole (LVM) demonstrated clinical benefit in a trial in patients with mild to moderate coronavirus disease 2019 (COVID-19), but its effect in a larger sample size needs to be confirmed.

\section{Methods}

In this randomized open-label trial, we enrolled non-hospitalized patients with mild to moderate COVID-19 at nine health centers in Tehran province, Iran, in 2021. Patients were randomly assigned to receive a 10day course of LVM with standard care $(n=185)$, or standard care $(n=180)$ in a 1:1 ratio. On days 1 to 10 , LVM was administered orally at a dosage of $50 \mathrm{mg}$. The participants were called and followed on days 1 , $3,5,7,9$, and 14 . The outcomes were general health condition, hospitalization rate, sign and symptoms, and adverse events (AEs). Generalized Estimating Equations model was used for analysis.

\section{Results}

Among 507 randomized patients, 473 started the experiment and received LVM in addition to standard care or received only the standard care (median age, 40 [IQR, 32-50.75] years; 164 [44.9\%] women; $9.4 \%$ had diabetes, $8.8 \%$ hypertension, $1.6 \%$ cardiovascular disease), and $346(98 \%)$ completed the trial. Compared to control group, LVM decreased the general health condition of the patients $(B=-0.635 ; \mathrm{Cl}=$ $-0.041,-0.329 ; P=0.000)$. Patients in the LVM with standard care group had significantly lower odds of developing fever $(\mathrm{OR}=0.260 ; 95 \% \mathrm{Cl}=0.113-0.599 ; \mathrm{P}=.002)$, chills $(\mathrm{OR}=0.223 ; 95 \% \mathrm{Cl}=0.076-0.648 ; \mathrm{P}=$ $0.006)$, fatigue $(\mathrm{OR}=0.576 ; 95 \% \mathrm{Cl}=0.346-0.960 ; \mathrm{P}=0.034)$, and myalgia $(\mathrm{OR}=0.544 ; 95 \% \mathrm{Cl}=$ $0.317-0.932 ; P=0.027)$. During the therapy, there was no significant difference in the parameters of dyspnea, cough, diarrhea, nausea, vomiting, sore throat, hyposmia, dysgeusia, and anorexia $(P>0.05)$. No significant difference was observed in the rate of hospitalization. Although the intervention group had greater AEs than the control group, yet, the difference was not statistically significant.

\section{Conclusions}

LVM has clinical benefit in improving health condition of patients with mild to moderate COVID-19. Further studies are needed to confirm our findings.

\section{Trial Registration:}

Iranian Registry of Clinical Trials: IRCT20201124049480N1; Registration date: 28/03/2021. 


\section{Introduction}

In late 2019, a new strand of coronaviruses was discovered in Wuhan, China. This virus caused a disease later named Coronavirus Disease 2019(COVID-2019), which initially seemed to have mostly respiratory manifestations(1). World Health Organization (WHO) announced the COVID-19 as a public health emergency of international concern (1). According to WHO statistics, to dat0065 (1 October 2021), COVID19 has infected approximately $233,503,524$ people worldwide and caused nearly $4,777,503$ deaths(2). According to WHO reports, Iran is among the 20 countries with the highest prevalence of COVID-19, and so far, 5,587,040 people have been infected, and 120,428 deaths have been reported due to COVID-19 in this country.

Almost no effective oral therapy exists for the outpatient treatment of coronavirus disease 2019 (COVID19). Reducing symptom severity and decreasing hospitalizations for outpatients is an important public health mitigation strategy for overcoming this pandemic. Levamisole (LVM) showed in vitro activity against severe acute respiratory syndrome coronavirus 2 (SARS-CoV-2) and has been proposed as a potentially effective treatment (3-6). Several recent clinical trials highlighted that LVM might be effective in preventing and treating SARS-CoV-2 infections but only one has been published (3)which was small in sample size and had methodological limitations, such as not removing the potential influence of time or the interactions (3). However, when taken early in the course of the disease, LVM may have therapeutic benefits in the treatment of mild to moderate disease. According to the national guideline for COVID-19 diagnosis and management $(7,8)$, mild COVID-19 symptoms include fever $<38^{\circ} \mathrm{C}$, sore throat with or without dry cough, chills, headache, anosmia, dysgeusia, nausea, vomiting, anorexia, diarrhea, myalgia, and fatigue. They may differ among individuals presenting with one or more of the symptoms. The vital signs (pulse rate, blood pressure, and respiration rate) are stable at this stage, and SpO2 (oxygen saturation level) is greater than $92 \%$. The moderate disease is attributed to the patients suffering from more server aforementioned symptoms besides respiratory symptoms (including shortness of breath, chest pain, and pressure, etc.) with or without fever $>38^{\circ} \mathrm{C}$, and $\mathrm{SpO} 2$ ranged from $90-93 \%$.

To our knowledge, no randomized clinical trials to date have investigated LVM for COVID-19 in nonhospitalized patients with large sample size. Moreover, LVM is a safe, low-cost, widely available drug. We hypothesized that starting LVM therapy during the first few days of symptom onset could alter the course of COVID-19 by reducing symptom severity and duration, as well as preventing hospitalizations. Our objective is to evaluate the ten days of LVM treatment added to the standard care compared with standard care on the clinical status on days $1,3,5,7,9,14$ following treatment initiation in COVID-19 patients with mild to moderate disease.

\section{Methods}

\subsection{Trial Design}


We conducted a 6month prospective, parallel, randomized, clinical trial on patients above 18 years old with mild to moderate COVID-19 who referred to nine COVID-19 selected health centers of Tehran and its suburbs from mid-April 2021 to mid-September 2021. Because management is most likely to be effective if given early in the disease course, we sought to enroll persons as soon as possible after symptom onset. The Ethics Committee of AJA University of Medical Sciences (Ethics ID: IR.AJA.REC.1399.199) and the Iranian Registry of Clinical Trials both gave their approval to this study (ID: IRCT20201124049480N1, first registration date: $28 / 03 / 2021$.). The full protocol is available on https://en.irct.ir/trial/54675. Written informed consent was obtained of all patients or legally authorized representatives.

\subsection{Setting and Participants}

We conducted this multicenter study in COVID-19 selected health centers from three health networks in Tehran ( South Tehran, Rey City, and Eslamshahr) including Akbarabad Health Center, Number 10 health center, Farmanfarmayan Health Center, Shahid Vahedi Health Center, Meysam Health Center from South Tehran Health Network; Shahid Yaghmaei Health Center from Rey City Health Network; Chahardangeh Health Center, Vavan health Center, Moosiabad Health Center, Ghaemieh Health Center from Eslamshar Health Network.

We included patients who satisfied the following criteria: non-hospitalized patients with either signs and symptoms indicating COVID-19 or spiral chest CT-scan indicating COVID19 or PCR-confirmed COVID-19 infection, aged 18 years or older, informed consent and acceptance of the patient or his companion for taking the drug, and had not taken LVM during the previous five days (due to the 16-hour half-life of the drug). We informed patients not to take medications that were not part of the study protocol, since they were candidates for outpatient care.

Patients were excluded from this trial if they met the following criteria: Negative RT-PCR for COVID-19, history of hepatitis, cirrhosis, or severe liver disorders, severe renal failure (estimated glomerular filtration rate less than $30 \mathrm{~mL} / \mathrm{min}$ ), shortness of breath due to cardiogenic pulmonary edema, taking LVM for any other reason (e.g., parasitic infections), history of allergic reaction or known allergy to LVM or any hypersensitivity reaction to LVM, history of cancer chemotherapy, lactating or pregnant women or planning to get pregnant within 30 days of the trial.

COVID-19 was diagnosed based on the findings of PCR test (using the real-time PCR technique with Pishtazteb kit from the Pishtazteb company in Iran). However, due to the highest efficacy of outpatient management of COVID19 (i.e., Levamisole) in the first four days of onset of symptoms, we sought to enroll patients as soon as possible after symptom onset. Furthermore, COVID-19 can be undetectable when symptoms first appear. The median false-negative rate of PCR testing was found to be 38 percent on the first day of symptom onset (range, 18 percent to 65 percent), decreasing during the subsequent days $(9,10)$. To overcome these challenges and initiate therapy as early as possible, we enrolled individuals with laboratory-confirmed COVID-19 or COVID-19 - compatible symptoms and an epidemiologic link to contact with laboratory-confirmed COVID-19 instead of waiting for PCR results. The 
follow-up was continued unless the PCR result was negative. Patients who were hospitalized during the research had their vital status checked on a regular basis.

\subsection{Intervention}

Patients were randomly assigned in a 1:1 ratio into intervention or control groups by the permuted block randomization method. The control group received only the standard COVID-19 care based on national protocol (Hydroxychloroquine tablets $200 \mathrm{mg}$ twice daily for five days, acetaminophen tablets $500 \mathrm{mg}$ every 6 hours in case of fever, naproxen tablets 500 mg every 8 hours in case of myalgia, Famotidine 40 mg daily, Diphenhydramine syrup 10 ccs every 8 hours in case of sore throat and cough, etc.) (Table1). The intervention group received Levamisole $50 \mathrm{mg} /$ day for ten days in addition to the standard care. 
Table 1

Baseline Characteristics in a Study of the Effect of Levamisole on Non-Hospitalized Patients with Coronavirus Disease 2019 (COVID-19)

\section{Characteristics}

Control group

$(n=180)$

Intervention group $(n=185)$

Gender, No. (\%)

Male

$91(50.6)$

110 (59.5)

Female

89 (40.4)

75 (40.5)

Age, Median (IQR), y

41 (34-55)

37 (30-48)

$<20$ y, No. (\%)

$3(2.8)$

$6(4.2)$

20-29 y, No. (\%)

$12(11.1)$

24 (16.8)

$30-39$ y, No. (\%)

$28(25.9)$

45 (31.5)

$40-49$ y, No. (\%)

$24(22.2)$

$36(25.2)$

$50-59$ y, No. (\%)

$29(26.9)$

17 (11.9)

$60-69$ y, No. (\%)

$8(7.4)$

15 (10.5)

$\geq 70$ y, No. (\%)

4 (3.7)

$0(0)$

Body mass index, Mean (SD)

$24.49(3.63)$

$25.24(4.10)$

$<18.5 \mathrm{~kg} / \mathrm{m}^{2}$, No. $(\%)$

$2(1.9)$

$4(2.8)$

$18.5-24.9 \mathrm{~kg} / \mathrm{m}^{2}$, No. (\%)

$58(55.2)$

$69(47.6)$

$25-29.9 \mathrm{~kg} / \mathrm{m}^{2}$, No. (\%)

$39(37.1)$

$58(40.0)$

$\geq 30 \mathrm{~kg} / \mathrm{m}^{2}$, No. (\%)

$6(5.7)$

$14(9.7)$

Vital signs

Systolic Blood Pressure, Median (IQR), mmHg

Diastolic Blood pressure, Median (IQR), mmHg

Pulse Rate ( $\mathrm{n} / \mathrm{min})$, Mean (SD)

Respiratory Rate (n/min), Median (IQR)

Temperature $\left({ }^{\circ} \mathrm{C}\right)$, Median $(\mathrm{IQR})$

$120(110-120)$

$120(110-120)$

75 (70-80)

$75(70-80)$

$80.49(8.05)$

$82.43(11.60)$

$16(15-18)$

$16(14-18)$

37 (36-37)

$37(36-37))$

Mean of room-air 02 Sat, \%, Median (IQR)

$96(95-97)$

$96(95-97)$

Social History, No. (\%)

Cigarette Smoker

$7(3.9)$

7 (3.8) 


\begin{tabular}{|c|c|c|}
\hline Characteristics & $\begin{array}{l}\text { Control group } \\
(n=180)\end{array}$ & $\begin{array}{l}\text { Intervention group } \\
(n=185)\end{array}$ \\
\hline Hukkah Smoker & $3(1.7)$ & $5(2.7)$ \\
\hline Opium Consumer & $2(1.1)$ & $1(0.5)$ \\
\hline \multicolumn{3}{|c|}{ Co-existing Conditions, No. (\%) } \\
\hline Diabetes Meletus Type I & $1(0.6)$ & $1(0.5)$ \\
\hline Diabetes Meletus Type II & $19(10.6)$ & $13(7.0)$ \\
\hline Asthma & $2(1.1)$ & $2(1.1)$ \\
\hline COPD & $2(1.1)$ & $1(0.5)$ \\
\hline Hypertension & $16(8.9)$ & $16(8.6)$ \\
\hline Acute Coronary Syndrome & $4(2.2)$ & $2(1.1)$ \\
\hline Thalassemia & $0(0.0)$ & $1(0.5)$ \\
\hline Corticosteroids use & $1(0.6)$ & $0(0.0)$ \\
\hline Past COVID-19 Infection & $2(1.1)$ & $5(2.7)$ \\
\hline \multicolumn{3}{|c|}{ Concomitant medications, No. (\%) } \\
\hline Acetaminophen & $73(40.8)$ & $82(44.3)$ \\
\hline Naproxen & $103(57.5)$ & $118(63.8)$ \\
\hline Famotidine & $87(48.6)$ & $102(55.1)$ \\
\hline Diphenhydramine & $61(34.1)$ & $75(40.5)$ \\
\hline Dextromethorphan & $26(14.5)$ & $34(18.4)$ \\
\hline Bromhexine & $39(21.8)$ & $43(23.2)$ \\
\hline Dimenhydrinate & $8(4.5)$ & $10(5.4)$ \\
\hline Loperamide & $13(7.3)$ & $14(7.6)$ \\
\hline Hydroxychloroquine & $19(10.6)$ & $22(11.9)$ \\
\hline Multivitamins & $52(29.1)$ & 58 (31.4) \\
\hline Vit D 50000 & $43(24.0)$ & $49(26.5)$ \\
\hline Zinc & $73(40.8)$ & $82(44.3)$ \\
\hline Chlordiazepoxide & $14(7.8)$ & $18(9.7)$ \\
\hline Promethazine & $4(2.2)$ & $6(3.2)$ \\
\hline
\end{tabular}




\begin{tabular}{|lll|}
\hline Characteristics & $\begin{array}{l}\text { Control group } \\
(\mathbf{n = 1 8 0})\end{array}$ & $\begin{array}{l}\text { Intervention group } \\
(\mathbf{n = 1 8 5})\end{array}$ \\
\hline $\begin{array}{l}\text { Time from symptom onset to enrollment, Median (IQR), } \\
\mathrm{d}\end{array}$ & $1(1-2)$ & $1(1-3)$ \\
\hline Time from symptom onset to diagnosis, Median (IQR), d & $2(2-4))$ & $3(2-4)$ \\
\hline $\begin{array}{l}\text { Time from past Covid infection history and reinfection, } \\
\text { median (IQR), d }\end{array}$ & $178(178-178)$ & $365(300-365)$ \\
\hline
\end{tabular}

\subsection{Outcomes and Follow-up}

We used a predetermined checklist to collect both objective and subjective(self-reported) data which included demographic characteristics such as age(year), gender(male/female), body mass index (BMI) $\left(\mathrm{kg} / \mathrm{m}^{2}\right)$; the initial vital signs such as blood pressure (systolic/diastolic $\mathrm{mmHg}$ ), temperature(c), pulse rate(/min), respiratory rate(/min), 02 saturation (percent); past medical history; and social history including cigarette smoking, alcohol drinking, opium use, and drug abuse. The health care experts at the health centers were obliged to call the participants on days 1 (the medication start date), 3, 5, 7, 9, and 14 to assess medication adherence, AEs, presence and severity of COVID-19 symptoms, COVID-19 PCR result, laboratory test results, hospitalization, and vital status. If participants were hospitalized within 14 days, we continued to monitor their vital status after the research ended. Clinical symptoms included the general condition of the patient, dyspnea, cough, diarrhea, nausea, vomiting, myalgia, fatigue, headache, sore throat, rhinorrhea, fever; anosmia, dysgeusia, anorexia, and loss of consciousness. We used a 10point to assess the patient's overall status

The general condition of the participants' score was self-assessed using a 10-point verbal numeric scale (VNS) (0 to 10, with 1-point increments) with 0 indicating no symptoms and 10 indicating death. Those who died of COVID-19 related complications were assigned a severity score of 10 for any surveys missed up until the date of death. Patients were educated on VNS through telephone and were informed of their previous given scores on each follow-up day.

We collected medication-related adverse events with directed questioning on the most common AEs. Investigators called or texted the participants who did not respond to follow-up surveys, to ascertain outcomes from them or their designated third-party contacts. If this was unsuccessful, investigators searched their vital status in the Integrated Health Record System (so-called SIB).

Agranulocytosis, anemia, and thrombocytopenia are uncommon AEs of LVM. Therefore, we recommended physicians obtain a $\mathrm{CBC}$ for participants on days 1 and 14 to rule out such lab data abnormalities. However, since only 16 participants brought their lab data results, we didn't include them for analysis.

The health professionals were trained to immediately refer the patient to selected hospital centers in case of any of the following symptoms: (1) Increase in respiratory rates esp. > 24 times per minute/ 
progressive course of shortness of breath/difficulty breathing, chest pain, burning, or heaviness in the chest (2) Peripheral cyanosis (3) Changes in consciousness/drowsiness/confusion. Participants were advised to consult a physician at the selected comprehensive health center if they experienced any of the following symptoms to determine whether they require hospitalization or continue their treatment or they need to add supportive therapies: (1) exacerbation of cough or occurrence of productive cough; (2) persistence or exacerbation of fever above $38.5^{\circ} \mathrm{C}$ after five days; (3) severe diarrhea not responding to oral replacement therapy with water and electrolytes; and (4) severe anorexia.

\subsection{Study End Points}

The initial primary outcome was the self-reported general health condition of the participants measured by VNS on days $3,5,7,9,14$. Secondary outcomes were the presence of any clinical symptom at day 3,5,7,9,14, adverse events (AEs), hospitalization, and death.

\subsection{Sample Size}

Except for one clinical trial with 25 participants in each group, there is no complete study that can be used to calculate the sample size. However, Considering the general health condition measured by VNS as the primary outcome, the significant level of $\alpha=0.05$, power of $1-\beta=0.90$, the minimum detectable difference between two groups $=0.3$, and attrition rate $=10 \%$, the required sample size was calculated 180 in each group.

\subsection{Randomization}

In this study, patients were randomly assigned into two arms of the study by permuted block randomization method. Six quadruple blocks including $A A B B, A B A B, A B B A, B B A A, B A B A$, and $B A A B$ were determined, and a random number table was utilized to select one of the six predefined blocks for each of the four patients. According to the order specified in each block, two patients received treatment $A$ (treatment with LVM) and two patients received treatment B (treatment without LVM).

The appropriate number of vials of open-label study drugs were assigned to the patient. Sites did not have access to the randomization list and were unaware of the treatments sequence. At the health center, study medication was distributed according to the random number allocated to each participant. The research pharmacies held this list, and statisticians verified that the randomization sequence was followed.

\subsection{Statistical Analysis}

Before analyzing the data, quantitative variables in terms of normality were examined through Kolmogronov-Smirnov test. Mann-Whitney $\mathrm{U}$ test was used to compare the general health condition in the intervention group and control group. The presence of symptoms at each time point was assessed with the Chi-square/Fisher exact test.

Generalized estimating equations (GEE), was used to test possible differences in the general health condition of patients (measured by VNS) and clinical presentations (using age groups, and the baseline 
presentation as a covariate), assuming LVM and follow-up (when applicable) as fixed factors, with marginal distribution and considering the interaction between LVM prescription and follow-ups. Linear and binary logistic models were selected for the primary and secondary outcomes respectively. The Quasi-likelihood under Independence Model Criterion (QIC) and Corrected Quasi-likelihood under Independence Model Criterion (QICC) were used to choose between different correlation structures and considering various interactions. The structure that obtained the smaller QIC or QICC was better according to this criterion. The odds ratio and $95 \% \mathrm{Cl}$ for change in severity score from baseline between groups are presented. Analysis of death was not done because of the zero-event rate.

All the statistical analysis was conducted by SPSS software version 20 according to the intention-to-treat principle (that is, all participants with data are included in the analyses regardless of their medication status) with a 2-sided type I error using an a of 0.05 .

\subsection{Ethical Approval}

This study was approved by the Ethics Committee of AJA University of Medical Sciences with reference number IR.AJA.REC.1399.199.

\section{Results}

\subsection{Patients' Characteristics and Baseline Features}

Of 536 patients who consented and were assessed for eligibility, 507 underwent randomization and began the study: 237 started a 10-day course of LVM added to the standard care, 254 patients started a 10-day course of the standard care only (Figure 1). Of the 29 patients who were not randomized, 13 did not meet eligibility criteria, and 16 declined to participate. 34 randomized patients did not receive treatment: 13 withdrew consent, and 9 had protocol violations. During the follow-ups, 108 randomized patients were excluded from the study due to their negative COVID-19 PCR results (Figure 1).

Demographics and disease characteristics of the patients in the two groups showed no significant difference (Table 1). The mean age of the patients was $41.35 \pm 13.28$ years, and $55.1 \%$ of the patients were male. Overall, $9.4 \%$ had diabetes, $8.8 \%$ had hypertension, $1.6 \%$ of patients had cardiovascular disease, $1.1 \%$ had asthma, and $1.9 \%$ had past COVID-19 infection. At the screening phase, both groups had a median oxygen saturation of $96 \%$ while breathing room air (IQR, 95-97). The median time from symptom onset to study enrollment and diagnosis in both groups was 2 and 3 days respectively (IQR= 13 , and 2-4 respectively). Other than LVM, patients in the control and intervention groups were essentially identically administered medicines (Table 1).

\subsection{Efficacy of Levamisole Treatment}


The clinical improvement of individual patients in the intervention group was compared to the control group at each follow-up and is presented in Table 2 and Additional file 1. Table3, Figures 2 and 3 show the odds ratio of each of these symptoms, which was calculated using the generalized estimating equation in a linear or binary logistic model.

Table 2-Primary Outcome in a Study of the Effect of Levamisole on Non-Hospitalized Patients with Coronavirus Disease 2019 (COVID-19)

\begin{tabular}{|llll|}
\hline General Health Condition, Median (IQR) & Control group & Intervention group & P value \\
\hline Day 1 & $6(5-7)$ & $6(4-7)$ & 0.365 \\
\hline Day 3 & $5(4-6)$ & $4(3-5)$ & 0.001 \\
\hline Day 5 & $4(3-5)$ & $3(2-5)$ & 0.000 \\
\hline Day 7 & $3(2-4)$ & $2(1-4)$ & 0.000 \\
Day 9 & $2(1-3)$ & $1(0-2)$ & 0.000 \\
Day 14 & $1(0-2)$ & $0(0-1)$ & 0.000 \\
\hline
\end{tabular}

Table 3- The Odds Ratio of Clinical Presentation of Patients with COVID-19 Comparing Control and Intervention groups by Generalized Estimating Equations 


\begin{tabular}{|llll|}
\hline Clinical Presentation & OR & Cl95\% & P value \\
\hline Fever & 0.260 & $0.113-0.599$ & 0.002 \\
\hline Chills & 0.223 & $0.076-0.648$ & 0.006 \\
\hline Cough & 0.638 & $0.376-1.083$ & 0.096 \\
\hline Fatigue & 0.576 & $0.346-0.960$ & 0.034 \\
\hline Headache & 0.613 & $0.371-1.013$ & 0.056 \\
\hline Myalgia & 0.544 & $0.317-0.932$ & 0.027 \\
\hline Sore throat & 0.651 & $0.248-1.712$ & 0.384 \\
\hline Rhinorrhea & 0.285 & $0.100-0.816$ & 0.019 \\
\hline Dyspnea & 0.495 & $0.095-2.573$ & 0.403 \\
\hline Anorexia & 0.771 & $0.372-1.594$ & 0.482 \\
\hline Nausea & 1.034 & $0.358-2.986$ & 0.951 \\
\hline Vomiting & 0.193 & $0.021-1.810$ & 0.150 \\
\hline Diarrhea & 0.966 & $0.263-3.552$ & 0.959 \\
\hline Hyposmia & 0.845 & $0.345-2.070$ & 0.713 \\
\hline Dysgeusia & 0.824 & $0.358-1.900$ & 0.650 \\
\hline
\end{tabular}

LVM has decreased the general health condition of the patients comparing to the control group $(B=-0.635$; $\mathrm{Cl}=-0.041,-0.329 ; \mathrm{P}=0.000$ ) (Figure2). Moreover, as shown in Table 2, when comparing each follow-up, on days $3,5,7,9$, and 14 , patients who were randomized to the intervention group had significantly better general condition status than those randomized to the control group ( $P=0.000$ by Chi-square test). Only 6 patients of the study population had been hospitalized, with a median time of 7.5 days between symptom onset and admission (IQR, 7-10.25 days). Although the hospitalization rate was too low, there was no significant difference between the two groups $(P=0.444)$.

In the intervention group, patients had significantly lower odds of complaining from fever compared to the control group $(\mathrm{OR}=0.260 ; 95 \% \mathrm{Cl}=0.113-0.599 ; \mathrm{P}=0.002)$ (Table3). When comparing fever status on different days, we only found a significant difference in fever status on days 3 and $5(P<0.05$, Additional file 1). Similarly, the odds of complaining from chills was significantly lower in patients receiving LVM with standard care, comparing to the control group ( $\mathrm{OR}=0.223 ; 95 \% \mathrm{Cl}=0.076-0.648 ; \mathrm{P}=0.006)$ but no significant difference was found when comparing two groups on each follow-up day by chi-square (Additional file 1). 
Cough was less common in the intervention group than in the control group, but the difference was not significant $(\mathrm{OR}=0.638 ; 95 \% \mathrm{Cl}=0.376-1.083 ; \mathrm{P}=0.096)$. Patients in the intervention group, however, showed significantly better cough status on days 3 and 14 when compared to the control group $(P=$ 0.034 and 0.005 , respectively, by chi-square).

The intervention group had significantly lower odds of fatigue. $(\mathrm{OR}=0.576 ; 95 \% \mathrm{Cl}=0.346-0.960 ; \mathrm{P}=$ 0.034). On day 7 of follow-up, the intervention group reported less fatigability than the control group which was statistically significant $(P=0.044)$.

Patients in the intervention group had significantly lower odds of suffering from myalgia than the control group $(\mathrm{OR}=0.544 ; 95 \% \mathrm{Cl}=0.317-0.932 ; \mathrm{P}=0.027)$. This effect was found over follow-up on 7 th $(\mathrm{P}=$ 0.001) and 9th $(P=0.016)$ days after receiving the interventions which indicate a significant improvement in the LVM group compared to the standard care group. Patients receiving LVM also had significantly fewer odds of rhinorrhea than those receiving only the standard care $(\mathrm{OR}=0.285 ; 95 \% \mathrm{Cl}=0.100-0.816 ; \mathrm{P}$ $=0.019)$.

Patients in the intervention group reported fewer headaches over a follow-up on 7th ( $P=0.003), 9$ th $(P=$ 0.009 ), and 14th days than the control group. The odds of suffering from headaches were less in the intervention group but this effect was not statistically significant $(\mathrm{OR}=0.613 ; 95 \% \mathrm{Cl}=0.371-1.013 ; \mathrm{P}$ $=0.056)$.

The parameters of dyspnea, cough, diarrhea, nausea, vomiting, sore throat, hyposmia, dysgeusia, and anorexia were also assessed on days $1,3,5,7,9$, and 14 . Our findings showed no significant difference in these variables at baseline and during the treatment $(P>0.05)$ except for cough which significantly more patients in the intervention group reported coughs $(P=0.012)$; thereby we considered adjusting cough at baseline in the GEE model. Loss of consciousness was not reported in either group during the study period. All the parameters assessed, apart from their significance, were less than 1 ; only nausea was observed to have $\mathrm{OR}>1$ which was not statistically significant (Figure 3).

The number of hospitalized patients in the control and intervention groups was $4(2.2 \%)$ and $2(1.1 \%)$ respectively $(P=0.444)$. The mortality rate was not analyzed due to the zero number of events.

\subsection{Adverse Events}

No severe AEs were reported by patients in either group. AEs were experienced by only $6 \%$ of patients in the study which were mild and self-limited including stomachache $(n=9)$, metallic taste in the mouth $(n=7)$, vertigo $(n=2)$, skin rash $(n=1)$, and oral ulcer $(n=1)$ (Table 4). The AEs, although rare, were more observed in the intervention group than the control group but this difference was not statistically significant (Table 3). 
Table 4

Comparison of Adverse Events between Control and Intervention Groups

\begin{tabular}{|c|c|c|c|}
\hline Adverse Events & Control group $(n=180)$ & Intervention group $(n=185)$ & $P$ value \\
\hline \multicolumn{4}{|c|}{ Stomachache, No (\%) } \\
\hline Day 1 & $0(0.0)$ & $5(2.9)$ & 0.336 \\
\hline Day 3 & $1(1.8)$ & $8(4.7)$ & 0.455 \\
\hline Day 5 & $1(1.8)$ & $4(2.4)$ & 1.000 \\
\hline Day 7 & $0(0.0)$ & $0(0.0)$ & - \\
\hline Day 9 & $0(0.0)$ & $0(0.0)$ & - \\
\hline Day 14 & $0(0.0)$ & $0(0.0)$ & - \\
\hline \multicolumn{4}{|l|}{ Vertigo, No (\%) } \\
\hline Day 1 & $0(0.0)$ & $1(0.6)$ & 1.000 \\
\hline Day 3 & $0(0.0)$ & $2(1.2)$ & 1.000 \\
\hline Day 5 & $0(0.0)$ & $1(0.6)$ & 1.000 \\
\hline Day 7 & $0(0.0)$ & $0(0.0)$ & - \\
\hline Day 9 & $0(0.0)$ & $1(0.6)$ & 1.000 \\
\hline Day 14 & $0(0.0)$ & $1(0.6)$ & 1.000 \\
\hline \multicolumn{4}{|c|}{ Skin Rash, No (\%) } \\
\hline Day 1 & $0(0.0)$ & $0(0.0)$ & - \\
\hline Day 3 & $0(0.0)$ & $0(0.0)$ & - \\
\hline Day 5 & $0(0.0)$ & $1(0.6)$ & 1.000 \\
\hline Day 7 & $0(0.0)$ & $1(0.6)$ & 1.000 \\
\hline Day 9 & $0(0.0)$ & $1(0.6)$ & 1.000 \\
\hline Day 14 & $0(0.0)$ & $1(0.6)$ & 1.000 \\
\hline \multicolumn{4}{|c|}{ Metallic Taste in Mouth, No (\%) } \\
\hline Day 1 & $0(0.0)$ & $7(4.1)$ & 0.197 \\
\hline Day 3 & $0(0.0)$ & $7(4.1)$ & 0.196 \\
\hline Day 5 & $0(0.0)$ & $3(1.8)$ & 0.574 \\
\hline Day 7 & $0(0.0)$ & $0(0.0)$ & - \\
\hline Day 9 & $0(0.0)$ & $0(0.0)$ & - \\
\hline
\end{tabular}




\begin{tabular}{|llll|}
\hline Adverse Events & Control group $(\mathbf{n}=\mathbf{1 8 0})$ & Intervention group $(\mathbf{n = 1 8 5})$ & P value \\
\hline Day 14 & $0(0.0)$ & $0(0.0)$ & - \\
\hline Insomnia, No (\%) & & & \\
\hline Day 1 & $1(1.8)$ & $1(0.6)$ & - \\
\hline Day 3 & $0(0.0)$ & $0(0.0)$ & - \\
\hline Day 5 & $0(0.0)$ & $0(0.0)$ & - \\
\hline Day 7 & $0(0.0)$ & $0(0.0)$ & - \\
\hline Day 9 & $0(0.0)$ & $0(0.0)$ & 1.000 \\
\hline Day 14 & $0(0.0)$ & $0(0.0)$ & 1.000 \\
\hline Oral Ulcer, No (\%) & & $1(0.6)$ & 1.000 \\
\hline Day 1 & $0(0.0)$ & $1(0.6)$ & - \\
\hline Day 3 & $0(0.0)$ & $1(0.6)$ & - \\
\hline Day 5 & $0(0.0)$ & $0(0.0)$ & - \\
\hline Day 7 & $0(0.0)$ & $0(0.0)$ & $0(0.0)$ \\
\hline Day 9 & $0(0.0)$ & & - \\
\hline Day 14 & $0(0.0)$ & & \\
\hline
\end{tabular}

\section{Discussion}

Individuals who underwent LVM therapy for 10 days showed significantly improved clinical status on day 14 than those who received standard care in this clinical study of patients with mild to severe COVID-19. The difference in the clinical status on days 3,5,7,9 and 14 between the 10-day LVM and standard care groups was significant. We used a 10-point scale (VNS) to assess the overall health of the participants in our study as they were handled outpatient. Unlike hospitalized patients, our goal in mild to moderately ill COVID-19 outpatients are the health improvement and wholeness of individuals in his/her opinion; One may experience cough but in their point of view, cough is tolerable. Although this is a subjective measurement, it is valuable.

The odds of fever, chills, fatigue, myalgia, rhinorrhea were also found to be significantly lower in the intervention group. The difference in the occurrence of dyspnea, cough, diarrhea, nausea, vomiting, sore throat, hyposmia, dysgeusia, and loss of appetite was not significant.

The reported symptoms by previous trial on LVM(3) with 50 patients were the following: fever (88.0\%), cough (78.0\%), dyspnea (54.0\%), asthenia (18.0\%), headache (8.0\%), dizziness (6.0\%), nausea $(6.0 \%)$, and myalgia (6.0\%). The most common symptoms at the beginning of this trial were fatigue $(51.3 \%)$, myalgia 
(48.9\%), fever (48.7\%), cough (47.6\%), headache (36.5\%), dizziness (\%), and chills (30.9\%), sore throat (30.1\%), anorexia (17.5\%), rhinorrhea (12.8\%), dysgeusia (12.8\%), hyposmia (12.5\%), nausea $(11.1 \%)$, diarrhea (9.2\%), dyspnea (8.6\%), vomiting (4.5\%).

The rate of hospitalization in our study was low (1.6\%), this is in agreement with the previous trial on LVM(3). Our multicenter trial took place in primary health centers, so we assumed that patients with a better overall health condition visited our study sites. On the other hand, COVID-19 suspected patients with severe health conditions would directly go to the hospitals, bypassing the health centers. Moreover, we only included COVID-19 patients who have a mild to moderate illness in our trial which could explain the low rate of hospitalization and mortality in our study.

The pro-inflammatory state is the second stage of COVID-19 illness, it is associated with an increased level of inflammatory cytokines such as IL-6 and IL-8 leading to cytokine storm, systemic inflammation, and severe acute respiratory syndrome (11). Therefore, researchers have focused on reducing inflammatory response as a potential therapeutic target against the second phase of COVID-19 disease. Many current researches are attempting to identify intracellular and molecular mechanisms as well as intervene to prevent COVID-19 illness from progressing to the second phase. In this regard, several studies evaluated the effect of LVM by explaining the molecular mechanisms. Arya et al. (7), in silico study, confirmed that LVM has a potential inhibitory effect on the Papaine Like Protease of the shell of the virus (which is necessary for virulence of COVID-19), can decrease the levels of TNF a and IL-6, and as a chemical adjutant, can introduce the virus to the immune system and might help manage COVID-19(6). Moreover, it was reported that LVM has an immune-enhancing effect, thereby increasing host immune response and viral clearance. Furthermore, Al-Kuraishy et al declared that co-administration of LVM with the COVID-19 vaccine may enhance the humoral immune response and immunization against SARS-CoV2(5).

So far, few specific antiviral and immunomodulatory treatments are available for COVID-19. Antiinflammatory and immunomodulatory treatments have been proven in studies to help manage COVID-19 patients who are in the pro-inflammatory stage of the disease (12). In severe cases of COVID-19, IL-6 levels in the blood are notably high [26]. Using Tocilizumab (TCZ), a human IL-6 blocking Medication, reduced C-reactive protein, oxygen demand, opacity of the lung lesion, and normalized the number of lymphocytes in $84.2,75,90.5$, and 52.6 percent of COVID-19 patients, respectively (13). Another anti-IL-6 agent is LVM. This medication inhibits IL-6's pro-inflammatory action and COVID-19 patients may benefit from this treatment.

With the same thought, several clinical trials on LVM in COVID-19 patients have been registered(1419) but the results are not yet published. However, in 2021, one trial published its results. Unlike the previous study (3) which found a significantly better cough status on days 3 and 14 in patients randomized to the LVM group, we found no significant difference in reducing cough between the two groups. We have to mention that our statistical analysis method was far more than only running a chisquare in SPSS to remove the effect of time and other probable interactions. Therefore, apart from chi- 
square which showed no difference in each follow-up comparison, we used a GEE model in the current study and confirmed that there is no difference in the odds of developing cough between the two groups.

The previous trial reported no significant differences in fever status on days $1,3,7$, and 14 between the intervention and control groups which contradicts our findings. We found significantly lower odds of reporting fever and also chills in the intervention group. The odds of not being feverish or not developing chills were almost 0.7 in the current study (Table 3 \& Figure 3 ). Moreover, on days 3 and 5 of follow-up, fever was less reported in the intervention group. Several factors may account for the lack of difference in the aforementioned outcomes observed in the 3-day LVM+ standard care group in the previous study. Given the low sample size of the previous study and the low dose of LVM continued for only three days, the actual antifebrile effect of LVM was not seen in the previous study.

Myalgia was also assessed in the previous study but unlike the current study (Table 3 \& Figure 3), they found no significant difference. Interestingly, only five (6\%) patients in the previous study were complaining of myalgia at the initial phase which is significantly fewer than the present study's 175 (48.9\%) patients. The discrepancy in findings between the two trials might be explained by the prior study's limited sample size and the inability to detect an efficacy outcome difference. Also, it might have happened since the previous study was conducted in 2020 with different variants of COVID-19 and less tendency of the virus to cause myalgia.

The previous trial reported significant differences between two groups in dyspnea after 7 and 14 days of follow-up, whereas the current study found no difference comparing days $3,5,7,9$, and 14 of follow-ups (Additional file 1). Although the odds of suffering from dyspnea were lower in the current study's intervention group, this finding did not have statistical significance (Table 3 \& Figure 3 ). Comparing the baseline dyspnea between the two studies, the percentage of patients with dyspnea in the previous study $(n=27,54.0 \%)$ is higher than in the current study $(n=31,8.6 \%)$. This might have happened due to the methodology and difference in inclusion criteria; so that patients with a poorer health condition were included in the previous study in contrast to the current study, that wide range of patients with 02 sat $>92 \%$ including both mild and moderate conditions with or without dyspnea were included.

Only a few AEs were reported in our trial which is congruent with the previous study (Table 4). However, further studies with larger sample size needs to confirm our findings.

\section{Limitation}

This trial has several limitations. First, COVID-19RT-PCR diagnostic testing was limited, non-hospitalized patients were often ineligible for testing, and the turnaround time for results was multiple days. second, the relatively low sample size in this trial made it impossible to calculate hospital length of stay, ICU admission, and mortality. Future trials should consider studying mortality and hospitalization rate in a higher study population. Third, because the patients had several coexisting diseases and were subjected to a diverse medication regimen, the results could have been affected by the heterogeneity of the sample and its treatment. Fourth, the patients were given a dose of only 50mg LVM after symptom onset to 
minimize the risk of AEs and better tolerance. The last limitation is that laboratory variables that could be used in identifying additional predictors of patients' outcomes were not collected. More objective methods such as broad laboratory evaluation that contribute to distinguishing patients progressing to severe and critical COVID-19 in both groups remain to be elucidated.

Further studies should determine whether preventive or early LVM prescription could be useful in the treatment of patients with COVID-19, especially those with mild or moderate disease.

\section{Conclusion}

Among patients with mild to moderate COVID-19, those randomized to a 10-day course of LVM added to the standard care had a statistically significant difference in general health status compared with standard care at 14 days after initiation of treatment. Patients randomized to a 10-day course of LVM added to the standard care had statistically significant odds of not developing a fever, chills, fatigue, myalgia, rhinorrhea compared with standard care at 14 days after initiation of treatment.

\section{Declarations}

\section{Acknowledgments}

We thank all the medical team at the health centers including doctors, nurses, the health care experts, and the staff who cooperated with this trial. We thank Dr. Jafar Sadegh Tabrizi, Dr. Namdaritabar, Dr. Mojgan Anvari, Dr. Ali Nikfarjam, Dr. Jamshid Motamed, Dr. Zahra Neda, and Dr. Najmeh Amini in the Ministry of Health and Education for facilitating the implementation of the study in the health centers. We thank Dr. Nahid Zomorrodin, Dr. Maryam Vali, Dr. Fatemeh Nasri, Dr. Mahboobe Ghanbarian, Dr. Mehdi Jalali, Dr. Mehran Eskandari, Farzaneh Ashouri, and Forough Shahrabi Farahani for visiting the patients and completing their follow-ups. We also thank the patients and their families for their cooperation.

\section{Authorship}

All named authors meet the International Committee of Medical Journal Editors (ICMJE) criteria for authorship for this article, take responsibility for the integrity of the work as a whole, and have given their approval for this version to be published

\section{Author contributions}

All authors contributed to the study conception and design. Material preparation and data collection were performed by Ali Zare Dehnavi, Behzad Khodaei and Atefeh Behkar. The design of the work was performed by Hamid Emadi Koochak, Ahmad Reza Dehpour, Hossein Khalili, and Mohammad Aminianfar. Analysis was performed by Mohammad Hassan Kazemi-Galougahi. The first draft of the 
manuscript was written by Mohammad Hossein Asgardoon and all authors commented on previous versions of the manuscript. All authors read and approved the final manuscript.

\section{Funding}

The manuscript was supported by AJA University of Medical Sciences with the grant number (IR.AJA.REC.1399.199), Tehran, Iran.

\section{Disclosures}

The authors declare that there is no conflict of interest regarding the publication of this article.

\section{Compliance with ethics guidelines}

This study was approved by the Ethics Committee of AJA University of Medical Sciences with reference number IR.AJA.REC.1399.199. This study was performed in accordance with the Helsinki Declaration of 1964, and its later amendments. All subjects provided informed consent to participate in this study.

\section{Consent for publication}

Not applicable.

\section{Data availability}

The datasets analyzed during the current study are available from the corresponding author on reasonable request.

\section{References}

1. Team EE. Note from the editors: World Health Organization declares novel coronavirus (2019-nCoV) sixth public health emergency of international concern. Eurosurveillance. 2020;25(5):200131e.

2. Organization WH. WHO Coronavirus (COVID-19) Dashboard. 2021.

3. Firozabad AR, Meybodi ZA, Mousavinasab SR, Sahebnasagh A, Jelodar MG, Karimzadeh I, et al. Efficacy and safety of Levamisole treatment in clinical presentations of non-hospitalized patients with COVID-19: a double-blind, randomized, controlled trial. BMC Infectious Diseases. 2021;21(1):18.

4. Uyaroğlu OA, Güven GS, Güllü İ. Can Levamisole be used in the treatment of COVID-19 patients presenting with diarrhea? The Journal of Infection in Developing Countries. 2020;14(08):844-6. 
5. Al-Kuraishy HM, Al-Gareeb Al, Alkazmi L, Alexiou A, Batiha GE-S. Levamisole Therapy in COVID-19. Viral Immunology. 2021.

6. Arya R, Das A, Prashar V, Kumar M. Potential inhibitors against papain-like protease of novel coronavirus (COVID-19) from FDA approved drugs. 2020.

7. Iranian quideline for COVID-19 diagnosis and management. 2021.

8. Rahmanzade R, Rahmanzadeh R, Hashemian SM, Tabarsi P. Iran's Approach to COVID-19: Evolving Treatment Protocols and Ongoing Clinical Trials. Frontiers in Public Health. 2020;8(523).

9. Kucirka L, Lauer S, Laeyendecker O, Boon D, Lessler J. Variation in false negative rate of RT-PCR based SARS-CoV-2 tests by time since exposure.

10. Cheng MP, Papenburg J, Desjardins M. Original: diagnostic testing for severe acute respiratory syndrome-related. N Engl J Med. 2020;382:727-33.

11. Lee C, Choi WJ. Overview of COVID-19 inflammatory pathogenesis from the therapeutic perspective. Archives of pharmacal research. 2021:1-18.

12. Alijotas-Reig J, Esteve-Valverde E, Belizna C, Selva-O'Callaghan A, Pardos-Gea J, Quintana A, et al. Immunomodulatory therapy for the management of severe COVID-19. Beyond the anti-viral therapy: A comprehensive review. Autoimmunity reviews. 2020;19(7):102569.

13. Xu X, Han M, Li T, Sun W, Wang D, Fu B, et al. Effective treatment of severe COVID-19 patients with tocilizumab. Proceedings of the National Academy of Sciences. 2020;117(20):10970-5.

14. Sciences YUoM. Evaluation of Levamisole efficacy and safety in combination with routine therapy in patients with Covid-19: a clinical trial. 2020.

15. Sciences SUoM. Assessment of effect of levamisole on pneumonia caused by COVID-19. 2020.

16. Company Npp. Evaluation of efficacy of pharmacotherapy treatment of COVID- 19 infection using oral Levamisole and Formoterol+Budesonide inhaler and comparison of this treatment protocol with standard national treatment of the disease. 2020.

17. (2020). ASUFSEE. Levamisole and isoprinosine in immune-prophylaxis of Egyptian healthcare workers facing COVID-19. Accessed: 5th May, 2020.

18. (2020). CUMED. Levamisole and isoprinosine in the treatment of COVID19: A proposed therapeutic trial. Accessed: 13th May, 2020.

19. (2020). FUoMSSA. Evaluation of efficacy of levamisole and formoterol+budesonide in treatment of COVID-19. Accessed: 13th April, 2020.

\section{Figures}




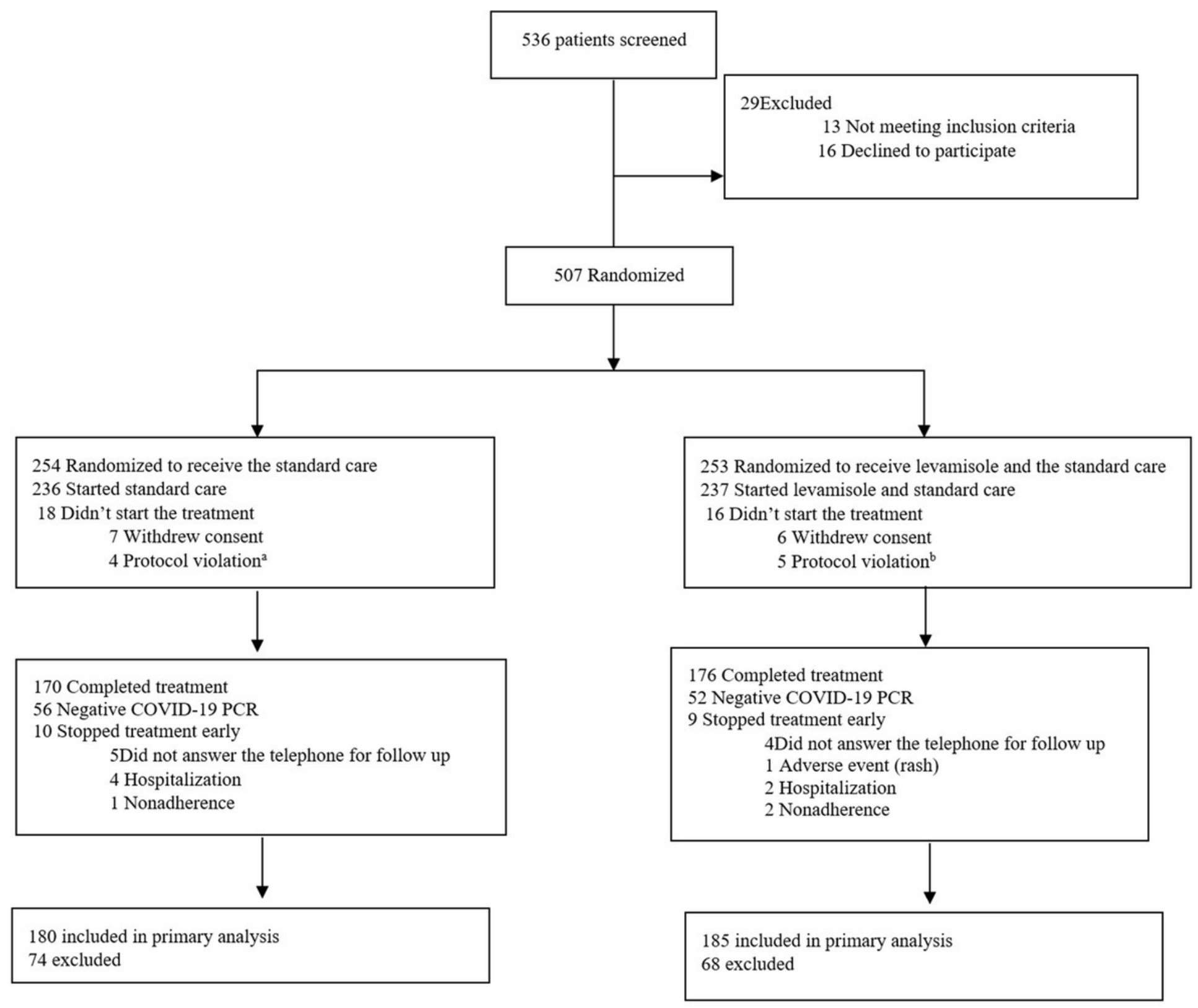

a. One patient had elevated liver enzymes, and one was pregnant.

b. Three patients were lactating, one was pregnant, and one had low GFR.

Figure 1

CONSORT 2010 Flow Diagram 


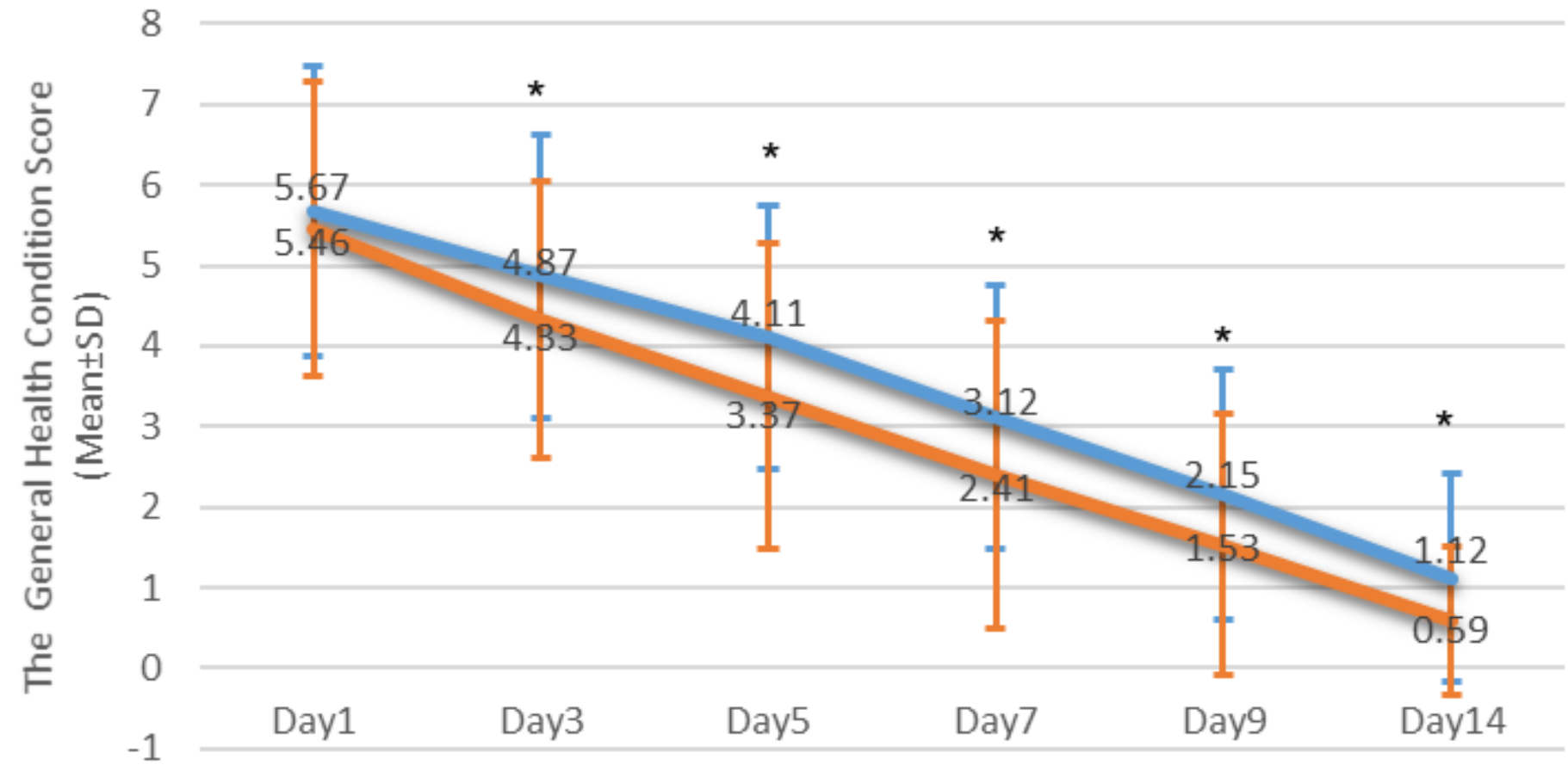

Time

Control Intervention

\section{Figure 2}

The General Health Condition of the Patients Measured by Visual Analogue Scale. The mean $\pm S D$ subjective overall condition of the patients on 6 follow-up times is shown. Significance is indicated by * for $\mathrm{P}<0.001$.

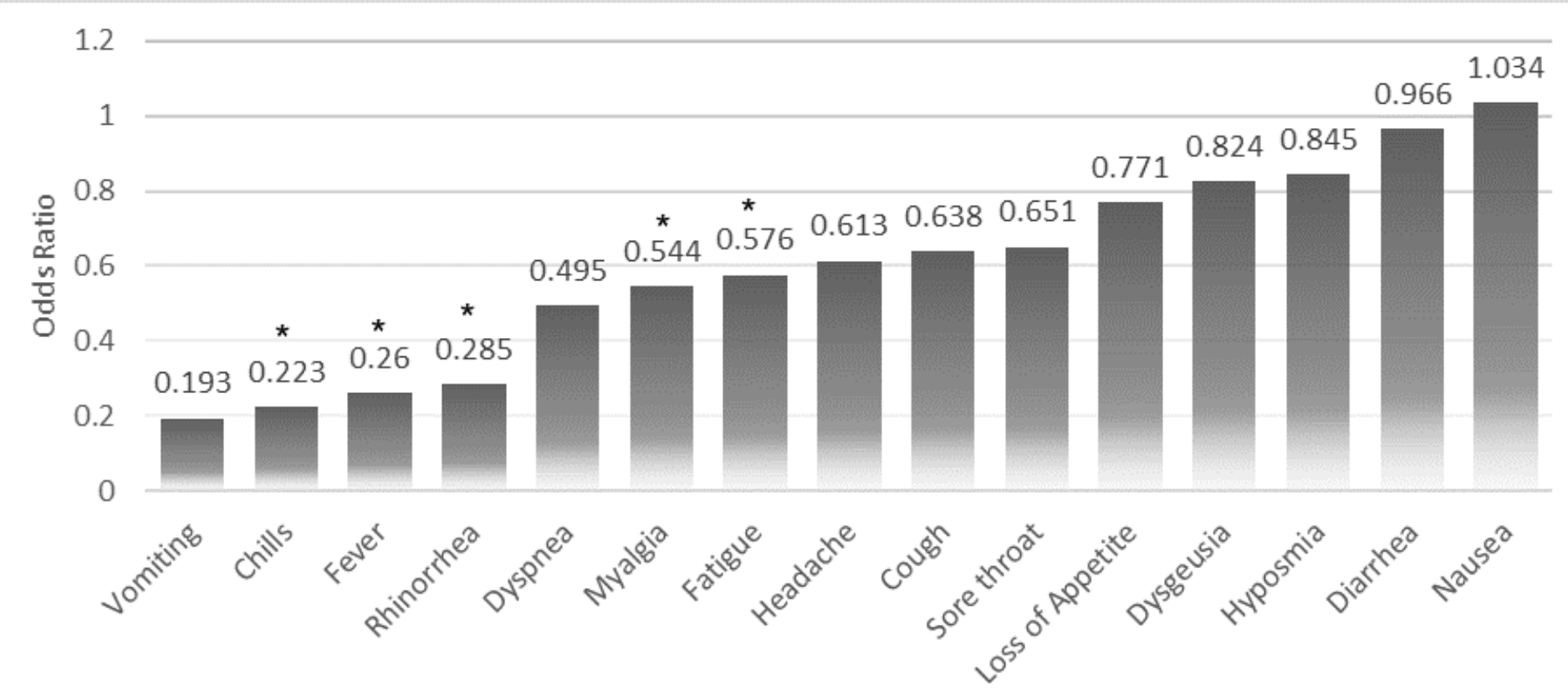

Clinical Presntations 


\section{Figure 3}

The Odd Ratio Of COVID-19 Related Symptoms in the Patients Receiving Levamisole and Standard Care Comparing to the Patients Receiving only Standard Care. The odds ratios presented were measured by a generalized estimating equation. $\mathrm{P}<0.05$ is indicated by *.

\section{Supplementary Files}

This is a list of supplementary files associated with this preprint. Click to download.

- SupplementaryFile1.docx 